\title{
REVISED Software for web-based tic suppression training [version
}

\section{2; referees: 3 approved]}

\author{
Jonathan K. Black ${ }^{1}$, Kevin J. Black (iD)2
}

${ }^{1}$ Ira A. Fulton College of Engineering and Technology, Brigham Young University, Provo, UT, 84602, USA

${ }^{2}$ Washington University School of Medicine, St. Louis, MO, 63110, USA

v2

First published: 19 Dec 2017, 6:2150 (doi: 10.12688/f1000research.13460.1)

Latest published: 06 Aug 2018, 6:2150 (doi: 10.12688/f1000research.13460.2)

Abstract

Exposure and response prevention (ERP) is a first-line behavior therapy for obsessive-compulsive disorder and Tourette syndrome (TS). However, ERP for tic disorders requires intentional tic suppression, which for some patients is difficult even for brief periods. Additionally, practical access to behavior therapy is difficult for many patients, especially those in rural areas. The authors present a simple, working web platform (TicTrainer) that implements a strategy called reward-enhanced exposure and response prevention (RE-ERP). This strategy sacrifices most expert therapist components of ERP, focusing only on increasing the duration of time for which the user can suppress tics through automated differential reinforcement of tic-free periods (DRO). RE-ERP requires an external tic monitor, such as a parent, during training sessions. The user sees increasing digital rewards for longer and longer periods of successful tic suppression, similar to a video game score. TicTrainer is designed with security in mind, storing no personally identifiable health information, and has features to facilitate research, including optional masked comparison of tics during DRO vs. noncontingent reward conditions. A working instance of TicTrainer is available from https://tictrainer.com/

Keywords

behavior therapy, software, tic disorders, Tourette syndrome, reward

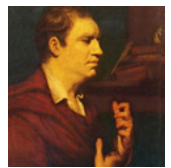

This article is included in the Tics collection.

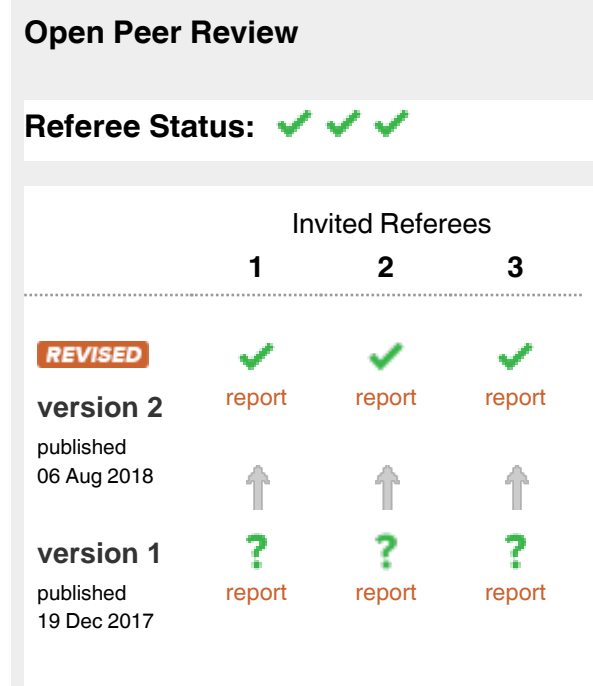

1 Christine A. Conelea, University of Minnesota, USA

2 Davide Martino, University of Calgary, Canada

3 Cara W.J Verdellen, PsyQ Nijmegen, Netherlands

Discuss this article

Comments (0) 
Corresponding author: Kevin J. Black (kevin@wustl.edu)

Author roles: Black JK: Software, Writing - Original Draft Preparation; Black KJ: Conceptualization, Resources, Writing - Original Draft Preparation, Writing - Review \& Editing

Competing interests: No competing interests were disclosed.

Grant information: The author(s) declared that no grants were involved in supporting this work.

Copyright: (C) 2018 Black JK and Black KJ. This is an open access article distributed under the terms of the Creative Commons Attribution Licence , which permits unrestricted use, distribution, and reproduction in any medium, provided the original work is properly cited.

How to cite this article: Black JK and Black KJ. Software for web-based tic suppression training [version 2; referees: 3 approved]

F1000Research 2018, 6:2150 (doi: 10.12688/f1000research.13460.2)

First published: 19 Dec 2017, 6:2150 (doi: 10.12688/f1000research.13460.1) 


\section{REVISED Amendments from Version 1}

The Introduction better describes evidence for traditional ERP. The Introduction and Conclusion highlight differences between traditional ERP and RE-ERP / TicTrainer.com. Previously the process of setting up an account and starting one's first training session were quite opaque, and the web site now shows improved "how to" instructions.

See referee reports

\section{Introduction}

Recent years have seen increasing evidence for and acceptance of behavior therapies for tic disorders such as Tourette syndrome (Capriotti et al., 2014). Tic suppression plays a key role in these therapies (Specht et al., 2014). One of these is exposure and response prevention (ERP), a first-line treatment for obsessivecompulsive disorder (Verdellen et al., 2011). In a randomized, controlled trial, ERP showed similar tic reduction efficacy to habit reversal therapy, the most extensively studied behavior therapy for tics, with a mean 8.6-point decrease in the Yale Global Tic Severity Scale (YGTSS) after 12 two-hour ERP sessions (Verdellen et al., 2004). However, tic suppression, an essential component of ERP, is difficult or frustrating for some tic patients. Fortunately, we showed that even children with recentonset tic disorders could suppress tics when brief tic-free periods were rewarded immediately by small tokens (Greene et al., 2015). Our experience with that study, and in an unrelated project, suggested to us that automating the process of immediate rewards for tic-free intervals might facilitate ERP, even in those who at first could suppress tics only for a few seconds at a time (Black et al., 2017; Miller et al., 2015). There is substantial evidence that contingent reward enhances tic suppression without producing a rebound effect, at least for periods of time up to 30-40 minutes (Brabson et al., 2016; Conelea et al., 2018; Himle et al., 2007; Himle et al., 2008; Specht et al., 2013; Woods et al., 2008). Within a single session, contingently reinforced tic suppression produced decreases in tic frequency whether or not the participants were directed to attend to premonitory urges (Specht et al., 2013). However, whether repeated practice of tic suppression leads to sustained, clinically relevant improvement is not known, because unlike typical ERP, tic suppression alone does not explicitly encourage attention to the premonitory urge (the "exposure" part of ERP).

Here we present a simple, web-based tool to facilitate training intended to allow increasing periods of tic suppression. Design goals included using this program to record tics, to provide rewards for tic suppression in a video-game-like format that many children would be familiar with, to gradually increase rewards for increasing periods of tic suppression, to respect confidentiality, to gather anonymous information that can be used to assess use patterns and initial information about efficacy and safety, and to provide features that facilitate research use, i.e. creation of research subject accounts that can be assigned to different reward schedules at different points in time.

\section{Methods}

Implementation

The TicTrainer server is written in JavaScript, using the Node.js runtime.

\section{Account registration and anonymity}

The system is designed to ensure security of users' personally identifying information. Instead of recording a name, each user is assigned a simple account ID used to $\log$ on. To track the collective age of users and to allow potential individualized training dependent on age, the user is asked his or her birth month and year. However, the system saves only a randomly chosen birthdate within 45 days of the 15th day of the month supplied.

Administrative accounts also can be created. Admins can flag certain users as research participants and assign them to receive rewards differently from regular users. They can also view system and session log files, or create another admin account.

\section{Storing data}

Each user and trainer account has its own text-based account data file on the server. This is a simple way to store modest amounts of data without using a database, at the possible expense of keeping numerous files open simultaneously if traffic to a single site becomes very heavy. System design may need to change if the number of users increases very substantially.

\section{Logging on}

A user's ID, password, and other account information are sent back and forth as needed between client and server. When a user types his ID and password into the "Manage Account" sign in page, for example, the server sends back a unique web page that includes the credentials, as he/she provided them, and some information loaded from his/her account data. If he/she edits something in his/her account, those same credentials are sent back to the server for authentication along with the new data.

Any page on the website that has information specific to a signed-in user is created dynamically by the server. These dynamic web pages are stored as .dynh files on the server. (".dynh" is a custom extension which stands for DYNamic Html.) These files have designated locations for the server to inject the required unique fields (usually these include the user's ID and password) before sending the edited page back to the client. Almost every page involved in a training session is stored and generated this way by the server, so as to preserve the user's credentials as he/she typed them at the beginning of the session.

During a session, the server mediates between the user and trainer via a session log file. When the trainer records a user's tic, the trainer's page sends an XMLHttpRequest asking the server to write a line to the log file. On the other side, the user's page continually checks the session log file on the server for changes, so it can reset the rewards when the user tics and end the session when the trainer leaves. At the end of a session, the log file is archived with the end time in the filename.

\section{Operation}

TicTrainer runs on a node.js server. Users need a web browser that supports javascript. The program may not function properly on browsers that do not support HTML5, and currently does not work with Microsoft browsers.

\section{Setting up}

A visitor to TicTrainer first registers an account. User and trainer accounts are created separately. Either one next goes 
to the "Manage Account" page and links to another account. Specifically, trainers specify the users they train, and users specify the trainers that can train them.

\section{Training sessions}

During a session, the trainer is presented with two buttons: "Tic Detected," and "End Session." When "Tic Detected" is pressed, the server logs a tic. Trainers also see a 1-minute timer progressing continuously next to an "I'm Here" button, which restarts that timer (as does the "Tic Detected" button). If the trainer presses "End Session," closes the page, or lets the "I'm Here" timer elapse, the session ends. The timer helps ensure that the trainer stays engaged in watching and recording the user's tics.

The user's session page displays a large counter for their current point total, followed by a superscript number indicating the current point accrual rate. For each session, the reward point rate starts at zero, and the rate resets to zero after each tic. Each time the user refrains from ticcing for a number of seconds equal to his/her level, their point total increases by the current rate, and the rate then increases by the square of the current level (rate is capped at $10 \times$ levels $^{2}$ ). Finally, a user "levels up" when his/her points exceed $1000 \times$ levels $^{2}$. He/she then also receives "coins" equal to the square of the previous level. The coins can be traded in for digital medals at an online store. Parents or clinicians may choose to provide tangible rewards for the digital coins or medals. The medals are displayed during training sessions and on the user's "Manage Account" page. In total, this reward strategy is intended to provide users increasing incentives to suppress their tics for increasingly long intervals.

For potential use in controlled trials, research participant accounts can also be assigned to an alternative (control) reward strategy, noncontingent reward (Greene et al., 2015; Himle et al., 2008).
In this case the admin user can set the initial mean frequency of rewards. This frequency may be set based on the participant's previously recorded tic frequency, to better mask the treatment allocation. Thereafter the frequency of rewards increases automatically based on the subject's achieved "level," with the intention of approximately matching reward frequency with the two methods.

\section{Use cases}

Figure 1 shows a "user" window (for the person with tics) and a "trainer" window as they might appear during a session. In typical use, the two windows would appear on two separate devices (but they can be opened on the same device, as shown here). This user is currently on level one, with 958 points, and is accruing 5 points for every second during which no tics are detected. This user has not yet earned any coins.

Supplementary File 1 is a session log file for a test session (no human subjects were observed). Admin users can view or download these session log files, from which they can compute for each session any of the following:

- $\quad$ measures of tic frequency and tic suppression, e.g.

$\circ$ mean frequency of tics

- longest tic-free interval

- number of 10 -second tic-free intervals

- number of rewards

- $\quad$ other metrics, e.g.

o tests of whether inter-tic intervals fit a fractal pattern (Peterson \& Leckman, 1998)

$\circ$ tests of the timing of tics vis-à-vis timing of rewards

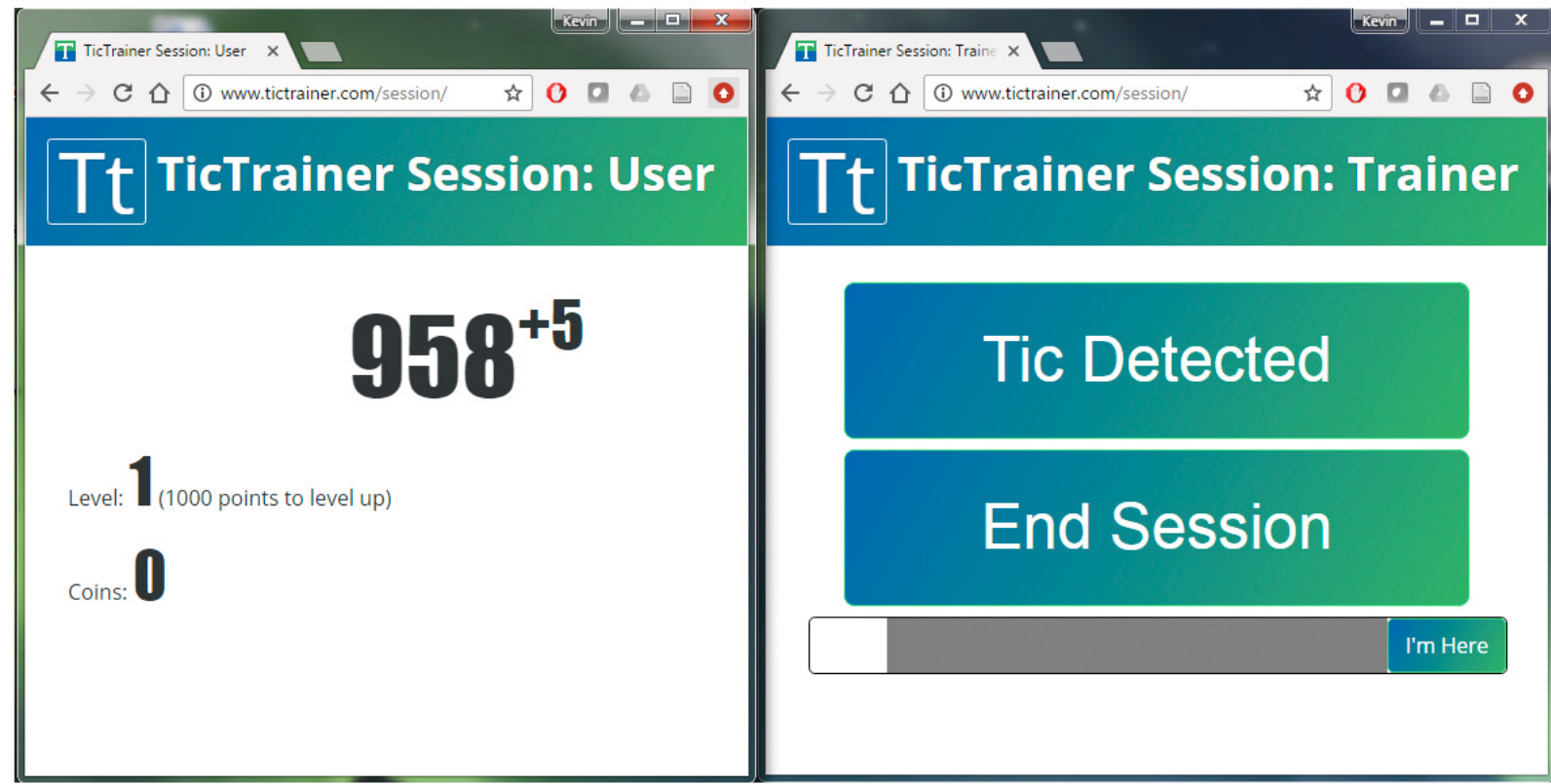

Figure 1. Screenshots from a TicTrainer.com session. Left: the "user" window (person with tics). Right: the "trainer" window (clinician or other trained observer). Typically the two windows would appear on separate devices. 


\section{Conclusions}

This simple web-based platform is available at TicTrainer.com, and provides features that allow prospective trials including with different reward schedules. Features that could yet be implemented include adding self-report (and/or trainer-report) measures of inter-session tic severity or of other symptoms, or measures of premonitory sensations/urge intensity before, during or after sessions (Brandt et al., 2016; Himle et al., 2007; Specht et al., 2014; Verdellen et al., 2008). Showing the user's personal record times (maximum achieved tic-free duration) would be another feature of interest.

Limitations of the software include the following. A "trainer" is required to monitor tics, yet neither machines nor humans are perfect tic monitors. The process of monitoring tics itself can reduce tic rate. The data recorded for the timing of tic occurrence does not distinguish motor vs. phonic tics, face vs. extremity tics, or simple vs. complex tics. This choice was in part driven by the senior author's experience monitoring tics for research, in that tracking individual tics separately in real time is impracticable for most patients. Additionally, while habit reversal therapy and its descendant Comprehensive Behavioral Intervention for Tics focus on one or two tics at a time, traditional ERP, like this software, focuses on tic suppression overall. The software itself does not encourage attention to premonitory urges, a focus of traditional ERP. One can provide instructions to do so outside of the software, or use the software without explicit exposure instructions in order to test whether ERP's efficacy depends on this component. Finally, this approach has not yet been tested for either tolerability or efficacy in tic patients.

\section{Software availability}

TicTrainer available from: https://tictrainer.com/

Source code available from https://github.com/jonkb/ TicTrainer-node

Archived source code as at time of publication: http://doi. org/10.5281/zenodo.1325945 (Black, 2018).

License: MIT

\section{Competing interests}

No competing interests were disclosed.

\section{Grant information}

The author(s) declared that no grants were involved in supporting this work.

\section{Acknowledgments}

A preprint of this work was posted on Authorea.com.

\section{Supplementary material}

Supplementary File 1: Sample regular log file. The log file from a sample training session. This test user account was not flagged for research, so this log file demonstrates the typical reward strategy (differential reinforcement of other behavior, or DRO).

Click here to access the data.

Black JK: TicTrainer V.3. Zenodo. 2018.

http://www.doi.org/10.5281/zenodo.1325945

Black JK, Koller JM, Black KJ: TicTimer software for measuring tic suppression [version 2; referees: 2 approved]. F1000Res. 2017; 6: 1560.

PubMed Abstract | Publisher Full Text | Free Full Text

Brabson LA, Brown JL, Capriotti MR, et al:: Patterned changes in urge ratings with tic suppression in youth with chronic tic disorders. J Behav Ther Exp Psychiatry. 2016: 50: 162-170.

PubMed Abstract | Publisher Full Text

Brandt VC, Beck C, Sajin V, et al:: Temporal relationship between premonitory urges and tics in Gilles de la Tourette syndrome. Cortex. 2016; 77: 24-37. PubMed Abstract | Publisher Full Text

Capriotti MR, Himle MB, Woods DW: Behavioral Treatments for Tourette Syndrome. J Obsessive Compuls Relat Disord. 2014; 3(4): 415-20. Publisher Full Text

Conelea CA, Wellen B, Woods DW, et al:: Patterns and Predictors of Tic Suppressibility in Youth With Tic Disorders. Front Psychiatry. 2018; 9: 188. PubMed Abstract | Publisher Full Text | Free Full Text

Greene DJ, Koller JM, Robichaux-Viehoever A, et al:: Reward enhances tic suppression in children within months of tic disorder onset. Dev Cogn Neurosci. 2015; 11: 65-74.

PubMed Abstract | Publisher Full Text | Free Full Text

Himle MB, Woods DW, Conelea CA, et al:: Investigating the effects of tic suppression on premonitory urge ratings in children and adolescents with Tourette's syndrome. Behav Res Ther. 2007; 45(12): 2964-76.

PubMed Abstract | Publisher Full Text

Himle MB, Woods DW, Bunaciu L: Evaluating the role of contingency in differentially reinforced tic suppression. J Appl Behav Anal. 2008; 41(2): 285-89. PubMed Abstract | Free Full Text

Miller B, Lim AN, Heidbreder AF, et al.: An Automated Motion Detection and Reward System for Animal Training. Cureus. 2015; 7(12): e397. PubMed Abstract | Publisher Full Text | Free Full Text

Peterson BS, Leckman JF: The temporal dynamics of tics in Gilles de la Tourette syndrome. Biol Psychiatry. 1998; 44(12): 1337-48.

PubMed Abstract | Publisher Full Text

Specht MW, Nicotra CM, Kelly LM, et al.: A Comparison of Urge Intensity and the Probability of Tic Completion During Tic Freely and Tic Suppression Conditions. Behav Modif. 2014; 38(12): 297-318.

PubMed Abstract | Publisher Full Text

Specht MW, Woods DW, Nicotra CM, et al.: Effects of tic suppression: ability to suppress, rebound, negative reinforcement, and habituation to the premonitory urge. Behav Res Ther. 2013; 51(1): 24-30.

PubMed Abstract | Publisher Full Text

Verdellen CW, Keijsers GP, Cath DC, et al: Exposure with response prevention versus habit reversal in Tourettes's syndrome: a controlled study. Behav Res Ther. 2004; 42(5): 501-11.

PubMed Abstract | Publisher Full Text 
Verdellen CW, Hoogduin CA, Kato BS, et al.: Habituation of premonitory sensations during exposure and response prevention treatment in Tourette's syndrome. Behav Modif. 2008; 32(2): 215-27.

PubMed Abstract | Publisher Full Text

Verdellen C, van de Griendt J, Hartmann A, et al.: European clinical guidelines for Tourette syndrome and other tic disorders. Part III: behavioural and psychosocial interventions. Eur Child Adolesc Psychiatry. 2011; 20(4): 197-207. PubMed Abstract | Publisher Full Text

Woods DW, Himle MB, Miltenberger RG, et al:: Durability, negative impact, and neuropsychological predictors of tic suppression in children with chronic tic disorder. J Abnorm Child Psychol. 2008; 36(2): 237-245.

PubMed Abstract | Publisher Full Text 


\section{Open Peer Review}

\section{Current Referee Status:}

\section{Version 2}

Referee Report 10 September 2018

doi:10.5256/f1000research.17288.r36851

\section{Davide Martino}

Department of Clinical Neurosciences, Cumming School of Medicine, Hotchkiss Brain Institute, University of Calgary, Calgary, AB, Canada

The authors' revisions have substantially improved the article and addressed my initial comments. I don't have further suggestions for improvement.

Competing Interests: No competing interests were disclosed.

I have read this submission. I believe that I have an appropriate level of expertise to confirm that it is of an acceptable scientific standard.

Referee Report 04 September 2018

doi:10.5256/f1000research.17288.r36852

\section{Cara W.J Verdellen}

PsyQ Nijmegen, Nijmegen, Netherlands

I have read the revisions with interest and noticed that the authors adequately addressed the questions raised.

Competing Interests: No competing interests were disclosed.

I have read this submission. I believe that I have an appropriate level of expertise to confirm that it is of an acceptable scientific standard.

\section{Referee Report 30 August 2018}

\section{doi:10.5256/f1000research.17288.r36853}

\section{Christine A. Conelea}

Department of Psychiatry, University of Minnesota, Minneapolis, MN, USA

The authors have been responsive to reviewer feedback and adequately addressed the questions raised. 
Competing Interests: No competing interests were disclosed.

Referee Expertise: Tic disorders, behavioral paradigms

I have read this submission. I believe that I have an appropriate level of expertise to confirm that it is of an acceptable scientific standard.

\section{Version 1}

Referee Report 15 March 2018

doi:10.5256/f1000research.14616.r30608

\section{Cara W.J Verdellen}

PsyQ Nijmegen, Nijmegen, Netherlands

TicTrainer is developed to support exposure and response prevention (ERP) in the behavioral treatment of tics. ERP is considered a firstline intervention for tic disorders according to European Guidelines (Roessner et al; Verdellen et al, ESSTS, 2011). Patients are encouraged to suppress tics for prolonged periods of time allowing them to get used to unpleasant premonitory urges that often accompany tics. TicTrainer is a very valuable, nice and simple tool that supports the patient in practicing ERP at home, also in the absence of a therapist. It is also based on the finding that tic suppression is enhanced by reinforcement of tic-free periods. TicTrainer stores anonymous information to assess use patterns and information about efficacy and safety. This makes TicTrainer a promising software tool for both clinical and research ends.

Unfortunately, I was unable to evaluate the website itself because after creating an account I did not manage to make it work ('Input Error: improper ID'). Therefore, my review is based only on the manuscript. I recommend the authors to pay attention to these "technical problems", and also to get it supported by Microsoft browsers, as this may be a major limitation for usage.

TicTrainer can be used to enhance tic suppression ability (response prevention of tics), but in its present form, it does not include the element of exposure to premonitory urges. Since exposure is an important part of ERP (learning to tolerate the urge, not give in to it by performing tics), it is highly recommended to integrate this element, for example by adding a measure of sensations/urges.

More literature demonstrating the effect of direct reinforcement on tic suppression should be included in the introduction as it supports the rational for this tool in enhancing tic suppression ability.

It is described in this manuscript that "preferably" two devices are needed; one for the patient and one for the trainer, who can be a parent/partner. It doesn't say if it also works on one device (do you see 2 windows?). A disadvantage of two devices may be that a patient always needs another person to practice with, which is not always possible. It is suggested to consider integrating both tic detection and suppression/reinforcement in one tool/device (like the app BT-Coach, see bt-tics.com/bt-coach).

For what age is this tool applicable?

Is it right that a patient doesn't see his tic suppression (record) times on the window, only "levels" and 
"earned coins"? It may be encouraging and reinforcing to also receive feedback on the exact tic suppression (record) times (as described in the protocol of ERP).

Since the tool is based on receiving rewards for tic suppression, perhaps more details can be given about what kind of rewards can be used; an example of a rewarding system could be useful. Perhaps "mini" games can be integrated in the tool that can be done directly after practicing, depending on the number of coins that are earned/levels achieved?

Overall I think this is a very promising tic suppression tool!

Is the rationale for developing the new software tool clearly explained?

Yes

Is the description of the software tool technically sound?

Partly

Are sufficient details of the code, methods and analysis (if applicable) provided to allow replication of the software development and its use by others?

Partly

Is sufficient information provided to allow interpretation of the expected output datasets and any results generated using the tool?

Partly

Are the conclusions about the tool and its performance adequately supported by the findings presented in the article?

Partly

Competing Interests: No competing interests were disclosed.

I have read this submission. I believe that I have an appropriate level of expertise to confirm that it is of an acceptable scientific standard, however I have significant reservations, as outlined above.

Author Response ( Member of the F1000 Faculty and F1000Research Advisory Board Member ) 02 Aug 2018

Kevin J Black, Department of Psychiatry, Washington University in St Louis, USA

TicTrainer is developed to support exposure and response prevention (ERP) in the behavioral treatment of tics. ERP is considered a firstline intervention for tic disorders according to European Guidelines (Roessner et al; Verdellen et al, ESSTS, 2011). Patients are encouraged to suppress tics for prolonged periods of time allowing them to get used to unpleasant premonitory urges that often accompany tics. TicTrainer is a very valuable, nice and simple tool that supports the patient in practicing ERP at home, also in the absence of a therapist. It is also based on the finding that tic suppression is enhanced by reinforcement of tic-free periods. TicTrainer stores anonymous information to assess use patterns and information about efficacy and safety. This makes TicTrainer a promising software tool for both clinical and research ends.

Unfortunately, I was unable to evaluate the website itself because after creating an account I did 
not manage to make it work ('Input Error: improper ID'). Therefore, my review is based only on the manuscript. I recommend the authors to pay attention to these "technical problems", and also to get it supported by Microsoft browsers, as this may be a major limitation for usage.

The web site is working now. Steps for registering and starting the first session were originally quite complicated. We now provide careful, step-by-step instructions for both "users" and "trainers," and we believe this also increases the likelihood of successful use.

TicTrainer can be used to enhance tic suppression ability (response prevention of tics), but in its present form, it does not include the element of exposure to premonitory urges. Since exposure is an important part of ERP (learning to tolerate the urge, not give in to it by performing tics), it is highly recommended to integrate this element, for example by adding a measure of sensations/urges.

In the edited introduction and conclusion, we now discuss this as a potential limitation, as a possible future improvement, and as an opportunity to test whether explicit exposure (focus on the urge) is essential to efficacy in ERP of tics (or whether by contrast tic suppression practice itself, if scheduled and repeated, can lead to benefit). This last possibility seems the more plausible given that habituation's relevance to ERP efficacy is now in doubt.

The relevant portions of the revised text include: "There is substantial evidence that contingent reward enhances tic suppression without producing a rebound effect, at least for periods of time up to 30-40 minutes (Himle et al., 2007; Himle et al., 2008; Woods et al., 2008; Specht et al., 2013; Brabson et al., 2016; Conelea et al., 2018). Within a single session, contingently reinforced tic suppression produced decreases in tic frequency whether or not the participants were directed to attend to premonitory urges (Specht et al., 2013). However, whether repeated practice of tic suppression leads to sustained, clinically relevant improvement is not known, because unlike typical ERP, tic suppression alone does not explicitly encourage attention to the premonitory urge (the "exposure" part of ERP).... The software itself does not encourage attention to premonitory urges, a focus of traditional ERP. One can provide instructions to do so outside of the software, or use the software without explicit exposure instructions in order to test whether ERP's efficacy depends on this component."

More literature demonstrating the effect of direct reinforcement on tic suppression should be included in the introduction as it supports the rational for this tool in enhancing tic suppression ability.

Done: "There is substantial evidence that contingent reward enhances tic suppression without producing a rebound effect, at least for periods of time up to 30-40 minutes (Himle et al., 2007; Himle et al., 2008; Woods et al., 2008; Specht et al., 2013; Brabson et al., 2016; Conelea et al., 2018). Within a single session, contingently reinforced tic suppression produced decreases in tic frequency whether or not the participants were directed to attend to premonitory urges (Specht et al., 2013)."

It is described in this manuscript that "preferably" two devices are needed; one for the patient and one for the trainer, who can be a parent/partner. It doesn't say if it also works on one device (do you see 2 windows?). A disadvantage of two devices may be that a patient always needs another 
person to practice with, which is not always possible. It is suggested to consider integrating both tic detection and suppression/reinforcement in one tool/device (like the app BT-Coach, see bt-tics.com/bt-coach).

We now address this question in "Use cases": "Figure 1 shows a "user" window (for the person with tics) and a "trainer" window as they might appear during a session. In typical use, the two windows would appear on two separate devices (but they can be opened on the same device, as shown here)."

We feel that BT-Coach is a wonderful (and more mature) application. However, the TicTrainer approach grew out of our recent study on children with recent onset of tics, who often are less likely to notice their own tics. We feel that tic monitoring by a third party may be a feature rather than a drawback in certain use cases.

For what age is this tool applicable?

We don't know for certain but expect that anyone who could sit through a session of even 10 minutes or so may be able to use it.

Is it right that a patient doesn't see his tic suppression (record) times on the window, only "levels" and "earned coins"? It may be encouraging and reinforcing to also receive feedback on the exact tic suppression (record) times (as described in the protocol of ERP).

Correct, though the increasing reward rate with increasing duration of tic suppression (through 10 seconds initially, or $10 \times$ level seconds later) is a weak proxy. We agree that the record times would be a potentially useful addition. We have added to conclusion the following: "Showing the user's personal record times (maximum achieved tic-free duration) would be another feature of interest."

Since the tool is based on receiving rewards for tic suppression, perhaps more details can be given about what kind of rewards can be used; an example of a rewarding system could be useful. Perhaps "mini" games can be integrated in the tool that can be done directly after practicing, depending on the number of coins that are earned/levels achieved?

That is a great idea that at this time is beyond our skill. One benefit of our releasing this code is that others with time and skill beyond ours can add to it.

Overall I think this is a very promising tic suppression tool!

Thank you!

Competing Interests: No competing interests were disclosed. 


\section{Davide Martino}

Department of Clinical Neurosciences, Cumming School of Medicine, Hotchkiss Brain Institute, University of Calgary, Calgary, AB, Canada

The concept of the TicTrainer is highly valuable. Unfortunately I was unable to review the current version of the TicTrainer website directly due to issues with registration (it won't accept ID - if crucial for this peer review, please provide some guidance in a Supplementary file or in the reply). In general, the authors should comment whether there is a plan in place to overcome the inability of Microsoft browsers to support the program, as this may be a major limitation for diffusion.

1. Like the other reviewer, I also think that the Introduction is too simplistic in comparing efficacy between ERP and CBIT, whereas in fact the quality of the evidence supporting these two behavioral treatments is different, and has been systematically reviewed and even meta-analysed. A short paragraph summarizing the evidence supporting the efficacy of ERP, also with respect to CBIT, would be useful.

2. Moreover, the Introduction should help a reader who is not fully acquainted with ERP understanding the technique of this behavioral approach, particularly the way in which trainer and patient interact during treatment sessions, and how this type of interaction is suitable to be operationalized using a software tool. All this is a bit given for well established in the paper, whereas in fact the literature on alternative modes of administering ERP is scant.

3. Perhaps I am missing some basic characteristic of the TicTrainer software, but from this manuscript it is not clear whether the "user" window and the "trainer" must appear on separate devices, or whether they could be opened on the same computer simultaneously. Can the authors comment on whether this is a specific choice of the authors of the software (and why), or a technical limitation that aims to be overcome in software upgrades?

4. Finally, I think that the lack of premonitory urge measures is an important limitation. On an individual patient basis, the lack of efficacy of contingent reward application to ERP might depend on the intensity of the premonitory urges and by an insufficient habituation to the urge. If the urges are not measured somehow, it would be difficult to monitor treatment response. A recent paper by Brandt et al., Cortex 2006 proposes a real time, computer-based urge monitoring technique; it would be interesting to integrate/implement something similar in this software.

Is the rationale for developing the new software tool clearly explained? Partly

Is the description of the software tool technically sound?

Partly

Are sufficient details of the code, methods and analysis (if applicable) provided to allow replication of the software development and its use by others?

Partly

Is sufficient information provided to allow interpretation of the expected output datasets and any results generated using the tool?

Partly 
Are the conclusions about the tool and its performance adequately supported by the findings presented in the article?

Partly

Competing Interests: No competing interests were disclosed.

I have read this submission. I believe that I have an appropriate level of expertise to confirm that it is of an acceptable scientific standard, however I have significant reservations, as outlined above.

Author Response ( Member of the F1000 Faculty and F1000Research Advisory Board Member ) 02 Aug 2018

Kevin J Black, Department of Psychiatry, Washington University in St Louis, USA

The concept of the TicTrainer is highly valuable. Unfortunately I was unable to review the current version of the TicTrainer website directly due to issues with registration (it won't accept ID - if crucial for this peer review, please provide some guidance in a Supplementary file or in the reply).

The web site is working now. Steps for registering and starting the first session were originally quite complicated. We now provide careful, step-by-step instructions for both "users" and "trainers," and we believe this also increases the likelihood of successful use.

In general, the authors should comment whether there is a plan in place to overcome the inability of Microsoft browsers to support the program, as this may be a major limitation for diffusion.

This is a quite reasonable concern, but we have not yet identified the specific problem. However, since other free browsers for MS Windows, Apple OS, Linux, Android and Chrome OS are in very common use, we suspect this will not constitute a real barrier to its use.

1. Like the other reviewer, I also think that the Introduction is too simplistic in comparing efficacy between ERP and CBIT, whereas in fact the quality of the evidence supporting these two behavioral treatments is different, and has been systematically reviewed and even meta-analysed. A short paragraph summarizing the evidence supporting the efficacy of ERP, also with respect to CBIT, would be useful.

I agree that the evidence for efficacy of ERP is less solid than for CBIT, but ERP advocates reasonably point to the Verdellen, 2004, trial comparing it to HRT, and their own experience. In any case, a long discussion seems out of place in a short paper introducing software. We added a citation in the introduction to the ESSTS review of behavior therapies for TS.

2. Moreover, the Introduction should help a reader who is not fully acquainted with ERP understanding the technique of this behavioral approach, particularly the way in which trainer and patient interact during treatment sessions, and how this type of interaction is suitable to be operationalized using a software tool. All this is a bit given for well established in the paper, whereas in fact the literature on alternative modes of administering ERP is scant. 
The introduction now points out that exposure by focusing on premonitory urges is an important part of traditional ERP and we discuss in introduction and conclusion how this approach differs.

3. Perhaps I am missing some basic characteristic of the TicTrainer software, but from this manuscript it is not clear whether the "user" window and the "trainer" must appear on separate devices, or whether they could be opened on the same computer simultaneously. Can the authors comment on whether this is a specific choice of the authors of the software (and why), or a technical limitation that aims to be overcome in software upgrades?

The original idea was for Johnny with tics to be seated at a computer or tablet and Mom to be using her smart phone, but it can be done on a single screen. We now specify this in "Use cases": "Figure 1 shows a "user" window (for the person with tics) and a "trainer" window as they might appear during a session. In typical use, the two windows would appear on two separate devices (but they can be opened on the same device, as shown here)."

4. Finally, I think that the lack of premonitory urge measures is an important limitation. On an individual patient basis, the lack of efficacy of contingent reward application to ERP might depend on the intensity of the premonitory urges and by an insufficient habituation to the urge. If the urges are not measured somehow, it would be difficult to monitor treatment response. A recent paper by Brandt et al., Cortex 2006 proposes a real time, computer-based urge monitoring technique; it would be interesting to integrate/implement something similar in this software.

We also are very interested in monitoring premonitory urge severity, though that likely would affect outcome. The conclusions now include the following: "Features that could yet be implemented include adding self-report (and/or trainer-report) measures of ... premonitory sensations/urge intensity before, during or after sessions (Brandt et al., 2016; Himle et al., 2007; Specht et al., 2014; Verdellen et al., 2008)."

Competing Interests: No competing interests were disclosed.

Referee Report 02 February 2018

doi:10.5256/f1000research.14616.r30166

Christine A. Conelea

Department of Psychiatry, University of Minnesota, Minneapolis, MN, USA

This paper describes TicTrainer, a newly created software program designed to track tic occurrences and deliver reward for successful suppression during live observation. Tic suppression contingent reward has reliably been shown to acutely enhance tic suppression in laboratory settings, but little work has been done to date to translate this finding into the clinical context. TicTrainer is an important step in this direction, and appears to be a promising tool for both research and clinical applications. To note, I was unable to evaluate the website itself due to "time out" errors, so my review is based only on the manuscript. Suggestions for enhancing the manuscript are as follows:

1. Introduction: In the sentence, "ERP also showed similar tic reduction.." clarify if the other treatment being referred to is Habit Reversal Therapy/Comprehensive Behavioral Intervention for Tics. It would be helpful to the reader to summarize the outcomes reported for ERP for tics in terms of 
response rate and degree of change in tic severity reported in prior research.

A key feature of ERP is that it aims to train tic suppression in the presence of the premonitory urge so that one can habituate to those aversive sensations. This should be noted in the introduction, particularly since this program would train suppression in a manner that is de-linked from the urge.

2. A brief summary of the literature demonstrating the robust, acute enhancement effect of contingent reward on tic suppression should be included in the introduction. This is a consistently replicated finding, which further supports the rationale for creating a more automated reward delivery program.

3. The introduction suggests an intent to utilize data input into the software for research purposes; however, at present the tictimer.com site does not appear to inform users of this intent. It is unclear to me if this is meant as a research only tool at this time, or if anyone can access the site. Clarify whether other possibility identifying information can be collected (e.g., IP address).

4. It seems that each "level" corresponds to a particular reinforcement schedule. Specific details about these schedules and how they change as one progresses through the program would be helpful. I assuming the program is intended to shape longer tic suppression durations but am not clear if the program aims to do this with an increasing or decreasing reward density. It also sounds like the reward schedule can be manipulated in a research context, so clarification of what the default settings vs. customizable settings would be useful.

5. A section discussing limitations of the current iteration of the software would be useful, such as reliance on live observation by the trainer (as tic frequency can be impacted by observation, and real-time judgements about tic occurrences can be difficult). The program only codes tic occurrence dichotomously, which precludes analyses related to tic type (e.g., motor vs. vocal, simple vs. complex).

6. It would be beneficial to clarify whether this software has been tested in those with tics yet.

Is the rationale for developing the new software tool clearly explained?

Yes

Is the description of the software tool technically sound?

Yes

Are sufficient details of the code, methods and analysis (if applicable) provided to allow replication of the software development and its use by others?

Yes

Is sufficient information provided to allow interpretation of the expected output datasets and any results generated using the tool?

Partly

Are the conclusions about the tool and its performance adequately supported by the findings presented in the article?

Partly 
Competing Interests: No competing interests were disclosed.

Referee Expertise: Tic disorders, behavioral paradigms

I have read this submission. I believe that I have an appropriate level of expertise to confirm that it is of an acceptable scientific standard, however I have significant reservations, as outlined above.

Author Response ( Member of the F1000 Faculty and F1000Research Advisory Board Member ) 02 Aug 2018

Kevin J Black, Department of Psychiatry, Washington University in St Louis, USA

This paper describes TicTrainer, a newly created software program designed to track tic occurrences and deliver reward for successful suppression during live observation. Tic suppression contingent reward has reliably been shown to acutely enhance tic suppression in laboratory settings, but little work has been done to date to translate this finding into the clinical context. TicTrainer is an important step in this direction, and appears to be a promising tool for both research and clinical applications. To note, I was unable to evaluate the website itself due to "time out" errors, so my review is based only on the manuscript.

The web site is working now. Steps for registering and starting the first session were originally quite complicated. We now provide careful, step-by-step instructions for both "users" and "trainers," and we believe this also increases the likelihood of successful use.

Suggestions for enhancing the manuscript are as follows:

Introduction: In the sentence, "ERP also showed similar tic reduction.." clarify if the other treatment being referred to is Habit Reversal Therapy/Comprehensive Behavioral Intervention for Tics. It would be helpful to the reader to summarize the outcomes reported for ERP for tics in terms of response rate and degree of change in tic severity reported in prior research.

Yes, the comparison was HRT/CBIT, which had more robust published evidence for efficacy. Since this is a software paper, the introduction was light on detail about ERP. Still, we now provide somewhat more information, as requested.

A key feature of ERP is that it aims to train tic suppression in the presence of the premonitory urge so that one can habituate to those aversive sensations. This should be noted in the introduction, particularly since this program would train suppression in a manner that is de-linked from the urge.

We now discuss this potentially important difference in the next-to-last paragraph of the introduction, and briefly mention it again at the end of the conclusion.

A brief summary of the literature demonstrating the robust, acute enhancement effect of contingent reward on tic suppression should be included in the introduction. This is a consistently replicated finding, which further supports the rationale for creating a more automated reward delivery program.

Done (next-to-last paragraph of introduction). 
1. The introduction suggests an intent to utilize data input into the software for research purposes; however, at present the tictimer.com site does not appear to inform users of this intent. It is unclear to me if this is meant as a research only tool at this time, or if anyone can access the site. Clarify whether other possibility identifying information can be collected (e.g., IP address).

Anyone can access the site and use the software as is (or create their own instantiation on their own server to change its details and control its use). The program is not intended to track IP address, but servers can do so.

2. It seems that each "level" corresponds to a particular reinforcement schedule. Specific details about these schedules and how they change as one progresses through the program would be helpful. I assuming the program is intended to shape longer tic suppression durations but am not clear if the program aims to do this with an increasing or decreasing reward density. It also sounds like the reward schedule can be manipulated in a research context, so clarification of what the default settings vs. customizable settings would be useful.

Higher levels give higher reward density but reward frequency is slower. We provide the details in the revised ms. ("For each session, the reward point rate starts at zero, and the rate resets to zero after each tic. Each time the user refrains from ticcing for a number of seconds equal to his/her level, their point total increases by the current rate, and the rate then increases by the square of the current level (rate is capped at $10 \times$ levels ${ }^{2}$ )." In other words, at level 1 , the first second without a tic is rewarded with 0 points, the 2nd second without a tic is rewarded by 1 point, and so on until the eleventh (and beyond) consecutive second without a tic is rewarded with 10 points. Once a tic happens, the rate resets to 0 point per second. At level 2 , two seconds must pass before the first reward, and the rate then increases to 4 points. After two additional seconds the rate increases to 8 points, and so on to a maximum of $\mathbf{4 0}$ points per 2 seconds. As for the question about research use, the current version of the software allows administrators to change a participant from the contingent reward schedule above to a noncontingent reward strategy in which rewards are given regardless of tics at a fixed initial rate that then increases with each subsequent level.

3. A section discussing limitations of the current iteration of the software would be useful, such as reliance on live observation by the trainer (as tic frequency can be impacted by observation, and real-time judgements about tic occurrences can be difficult). The program only codes tic occurrence dichotomously, which precludes analyses related to tic type (e.g., motor vs. vocal, simple vs. complex).

Done (last paragraph).

4. It would be beneficial to clarify whether this software has been tested in those with tics yet.

We're just offering the software. We don't yet know whether it will work or how well it is tolerated (though of course we want to find out). Added to last paragraph. 
Competing Interests: No competing interests were disclosed.

Author Response ( Member of the F1000 Faculty and F1000Research Advisory Board Member) 06 Aug 2018

Kevin J Black, Department of Psychiatry, Washington University in St Louis, USA

Oops, that sentence should have read:

"Higher levels give higher reward magnitude, but reward frequency is slower."

Competing Interests: No competing interests were disclosed.

The benefits of publishing with F1000Research:

- Your article is published within days, with no editorial bias

- You can publish traditional articles, null/negative results, case reports, data notes and more

- The peer review process is transparent and collaborative

- Your article is indexed in PubMed after passing peer review

- Dedicated customer support at every stage

For pre-submission enquiries, contact research@f1000.com 